\title{
Hypofractionated Stereotactic Radiation Therapy in Recurrent High-Grade Glioma: A New Challenge
}

\author{
Pierina Navarria, $\mathrm{MD}^{1}$ \\ Anna Maria Ascolese, MD ${ }^{1}$ \\ Stefano Tomatis, $\mathrm{PhD}^{1}$ \\ Giacomo Reggiori, $\mathrm{PhD}^{1}$ \\ Elena Clerici, MD ${ }^{1}$ \\ Elisa Villa, MD ${ }^{1}$ \\ Giulia Maggi, $\mathrm{MD}^{1}$ \\ Lorenzo Bello, MD² \\ Federico Pessina, $\mathrm{MD}^{2}$ \\ Luca Cozzi, PhD ${ }^{1}$ \\ Marta Scorsetti, MD
}

Departments of ${ }^{1}$ Radiotherapy and Radiosurgery and ${ }^{2}$ Neurosurgical Oncology, Istituto Clinico Humanitas Cancer Center and Research Hospital, Milano, Italy

Correspondence: Pierina Navarria, MD

Department of Radiotherapy and Radiosurgery, Istituto Clinico Humanitas Cancer Center,

via Manzoni 56, 20100-Rozzano, Italy

Tel: $39-2-8224-7458$

Fax: 39-2-8224-8509

E-mail: piera.navarria@cancercenter.humanitas.it

Received September 16, 2014

Accepted November 25, 2014

Published Online February 23, 2015

\begin{abstract}
Purpose
The aim of this study was to evaluate outcomes of hypofractionated stereotactic radiation therapy (HSRT) in patients re-treated for recurrent high-grade glioma.
\end{abstract}

\section{Materials and Methods}

From January 2006 to September 2013, 25 patients were treated. Six patients underwent radiation therapy alone, while 19 underwent combined treatment with surgery and/or chemotherapy. Only patients with Karnofsky Performance Status (KPS) $>70$ and time from previous radiotherapy greater than 6 months were re-irradiated. The mean recurrent tumor volume was $35 \mathrm{~cm}^{3}$ (range, 2.46 to $116.7 \mathrm{~cm}^{3}$ ), and most of the patients (84\%) were treated with a total dose of 25 Gy in five fractions (range, 20 to 50 Gy in 5-10 fractions).

\section{Results}

The median follow-up was 18 months (range, 4 to 36 months). The progression-free survival (PFS) at 1 and 2 years was $72 \%$ and 34\% and the overall survival (OS) 76\% and 50\%, respectively. No severe toxicity was recorded. In univariate and multivariate analysis extent of resection at diagnosis significantly influenced PFS and OS $(p<0.01)$. Patients with smaller recurrent tumor volume treated had better local control and survival. Indeed, the 2-year PFS was $40 \%\left(\leq 50 \mathrm{~cm}^{3}\right)$ versus $11 \%(p=0.1)$ and the 2 -year 0 S $56 \%$ versus $33 \%$ (> 50 $\left.\mathrm{cm}^{3}\right)$, respectively $(\mathrm{p}=0.26)$.

\section{Conclusion}

In our experience, HSRT could be a safe and feasible therapeutic option for recurrent high grade glioma even in patients with larger tumors. We believe that a multidisciplinary evaluation is mandatory to assure the best treatment for selected patients. Local treatment should also be considered as part of an integrated approach.

\section{Introduction}

The current standard of care for newly diagnosed glioblastoma is maximal safe resection followed by radiation therapy (RT) in association with concomitant and adjuvant temozolomide (TMZ) [1]. Tumor recurrence, however, affects about $90 \%$ of patients. The median overall survival (OS) is 15-18 months and less than $10 \%$ of patients are alive at 5 years [2]. Long-term local or regional control is rarely achieved and the
Key words

Glioma, Radiosurgery, Retreatment 
Table 1. Patient characteristics and treatments

\begin{tabular}{|c|c|c|c|}
\hline Characteristic & GBM & Grade III & Total \\
\hline \multicolumn{4}{|l|}{ Sex } \\
\hline Female & $7(70)$ & $3(30)$ & 10 \\
\hline Male & $6(40)$ & $9(60)$ & 15 \\
\hline Median age (range, yr) & $50(43-75)$ & $42(23-81)$ & - \\
\hline \multicolumn{4}{|l|}{ MGMT promoter methylation status } \\
\hline Methylated & $3(42.9)$ & $4(57.1)$ & 7 \\
\hline Unmethylated & $2(66.7)$ & $1(33.3)$ & 3 \\
\hline Unknown & $8(53.3)$ & $7(46.7)$ & 15 \\
\hline \multicolumn{4}{|l|}{ IDH mutation } \\
\hline Present & $1(33.3)$ & $2(66.7)$ & 3 \\
\hline Absent & $2(66.7)$ & $1(33.3)$ & 3 \\
\hline Unknown & $10(52.6)$ & $9(47.4)$ & 19 \\
\hline \multicolumn{4}{|l|}{ 1p19q codeletion status } \\
\hline Codeleted & NA & 3 & 3 \\
\hline Non-codeleted & NA & NA & - \\
\hline Unknown & NA & NA & - \\
\hline \multicolumn{4}{|l|}{ Time to relapse from initial diagnosis (mo) } \\
\hline$\leq 12$ & $7(70)$ & $3(30)$ & 10 \\
\hline $12-24$ & $3(50)$ & $3(50)$ & 6 \\
\hline$>24$ & $3(33.3)$ & $6(66.7)$ & 9 \\
\hline \multicolumn{4}{|l|}{ Treatment at initial diagnosis } \\
\hline \multicolumn{4}{|l|}{ Surgery } \\
\hline Complete resection & $9(60)$ & $6(40)$ & 15 \\
\hline Subtotal resection & $1(100)$ & $0(0)$ & 1 \\
\hline Partial resection & $1(14.3)$ & $6(85.7)$ & 7 \\
\hline Biopsy & $2(100)$ & 0 & 2 \\
\hline Radiotherapy & $13(52)$ & $12(48)$ & 25 \\
\hline $\mathrm{CT}$ concomitant and adjuvant (TMZ) & $13(52)$ & $12(48)$ & 25 \\
\hline
\end{tabular}

Values are presented as number (\%). GBM, glioblastoma; MGMT, methylguanine-DNA methyltransferase; IDH, isocitrate dehydrogenase; NA, not acquired; $\mathrm{CT}$, chemotherapy; TMZ, temozolomide.

ous system (CNS) tissues are not able to fully repair earlier radiation injury. Recent studies on primates and initial limited clinical series have nevertheless shown a substantial recovery of critical CNS structures [14]. In fact, this evidence, along with the improvement of neuro-imaging, and the availability of modern high-precision radiotherapy techniques, allowed the introduction of re-irradiation in the clinical practice. Stereotactic RT has emerged as a feasible treatment technique for recurrent brain tumors that have been exposed to high doses of RT [15]. Compared with conventional RT, stereotactic techniques, given as single fraction radio-surgery (SRS), fractionated stereotactic RT (FSRT) or hypofractionated stereotactic RT (HSRT), allow a more accurate dose delivery with a rapid dose falloff towards critical structures. From a radiobiological standpoint, SRS with the delivery of single high-dose fractions could be advantageous since it increases the cell killing effect or cell division capability arrest regardless of the mitotic phase. On the other hand, only small tumors can be treated with SRS due to the high risk of symptomatic radio-necrosis. In some reports the observed radio-necrosis incidence was as great as $31 \%$ when large tumors were treated $[16,17]$. FSRT exploits the radiobiological advantage of fractionation while reducing severe side effects, and allows treatment of larger tumors and/or tumors in eloquent areas. In particular, HSRT has the advantages of reduced treatment time with consequent reduction of the patient discomfort, the radiobiological advantage of fractionation allows treatment of even large tumor volumes with low risk of acute or sub-acute toxicity, and finally reduced incidence of symptomatic radio-necrosis [18-21]. No randomized trials have been performed and no consensus exists about the optimal total dose and the fractionation schedule. The aim of this retrospective study was to evaluate efficacy and toxicity of HSRT in patients re-treated for recurrent high-grade glioma (HGG). 
Table 2. Treatments at recurrence

\begin{tabular}{|c|c|c|c|}
\hline Variable & GBM & Grade III & Total \\
\hline \multicolumn{4}{|l|}{ Treatment at recurrence } \\
\hline Combined modality & $12(63.2)$ & $7(36.8)$ & 19 \\
\hline Radiotherapy alone & $1(16.7)$ & $5(83.3)$ & 6 \\
\hline \multicolumn{4}{|l|}{ Radiotherapy } \\
\hline \multicolumn{4}{|c|}{ Median volume of recurrent disease } \\
\hline$<35 \mathrm{~cm}^{3}$ & $8(61.5)$ & $5(38.5)$ & 13 \\
\hline$\geq 35 \mathrm{~cm}^{3}$ & $5(41.7)$ & $7(58.3)$ & 12 \\
\hline \multicolumn{4}{|l|}{ Dose prescription } \\
\hline 25 Gy / 5 fractions & $13(54.1)$ & $11(45.9)$ & 24 \\
\hline 50 Gy / 10 fractions & 0 & 1 (100) & 1 \\
\hline \multicolumn{4}{|l|}{ Chemotherapy } \\
\hline Temozolomide rechallenge & $5(83)$ & $1(17)$ & 6 \\
\hline Bevacizumab & $1(100)$ & 0 & 1 \\
\hline ACNU & 0 & $1(100)$ & 1 \\
\hline Fotemustine & $7(70)$ & $3(30)$ & 10 \\
\hline PC scheme & 0 & $1(100)$ & 1 \\
\hline \multicolumn{4}{|l|}{ Surgery } \\
\hline Subtotal resection & $3(50)$ & $3(50)$ & 6 \\
\hline Complete resection & $2(40)$ & $3(60)$ & 5 \\
\hline
\end{tabular}

Values are presented as number (\%). GBM, glioblastoma; ACNU, adjuvant chemotherapy with nimustine; PC, paclitaxel and carboplatin.

\section{Materials and Methods}

\section{Patients and procedures}

The present retrospective study includes patients with magnetic resonance imaging (MRI) evidence of recurrent or progressive HGG, occurring at least 6 months after RT completion (to exclude pseudo-progression). Progression was defined using the Response Assessment in Neuro-Oncology (RANO) criteria [22]. At the initial diagnosis, all patients underwent open-surgery resection to maximally remove the tumor $(>95 \%)$. The extent of resection (EOR) was determined by comparing the MRI before surgery with the MRI acquired within 48-hour postsurgery. Complete resection (CR) was defined as residual tumor volume lower than 1.5 $\mathrm{cm}^{3}$, subtotal resection (SR) between 1.5 and $15 \mathrm{~cm}^{3}$ and partial resection (PR) greater than $15 \mathrm{~cm}^{3}$ [23]. Within 4 weeks of surgery all patients underwent adjuvant RT (60 Gy in 30 fractions) and concomitant and sequential TMZ following the Stupp scheme [1]. At the time of recurrence, they were evaluated for salvage treatment (including re-resection of the tumor when indicated, HSRT, chemotherapy, or combined approaches) based upon patient clinical conditions, tumor site and volume, and hematologic rescue. To define the appropriate therapeutic strategy, a multidisciplinary board including a medical oncologist, a radiation oncologist and a neurosurgeon evaluated each patient. For local approaches (surgery and RT), only patients with Karnofsky Performance Status (KPS) $>70$ and an interval time from previous surgery or radiotherapy over 6 months were considered appropriate.

From January 2006 to September 2013, among patients referred at our institution for recurrent HGG, 25 patients underwent HSRT and were included in the present evaluation. Fifteen $(60 \%)$ were male and $10(40 \%)$ female, with a median age of 50 years (range, 23 to 81 years). Thirteen patients $(52 \%)$ had a diagnosis of glioblastoma (GBM), and 12 patients $(48 \%)$ were affected by grade III glioma (anaplastic astrocytoma/anaplastic oligoastrocytoma). Characteristics and treatments of patients are shown in Table 1. To precisely define the tumor extension, enhanced T1-MRI, FLAIR-MRI sequences were acquired and integrated with [11C]MET positron emission tomography-computed tomography $(\mathrm{CT})$ in some patients. The procedure for target definition included enhanced contrast CT scans. Patients were placed in the supine position with arms close to the body. A personalized thermoplastic mask was used for better patient immobilization and repositioning. All scans, extending from the top of the skull to the third cervical vertebrae, were acquired with 1-3-mm slice thickness and were transferred 

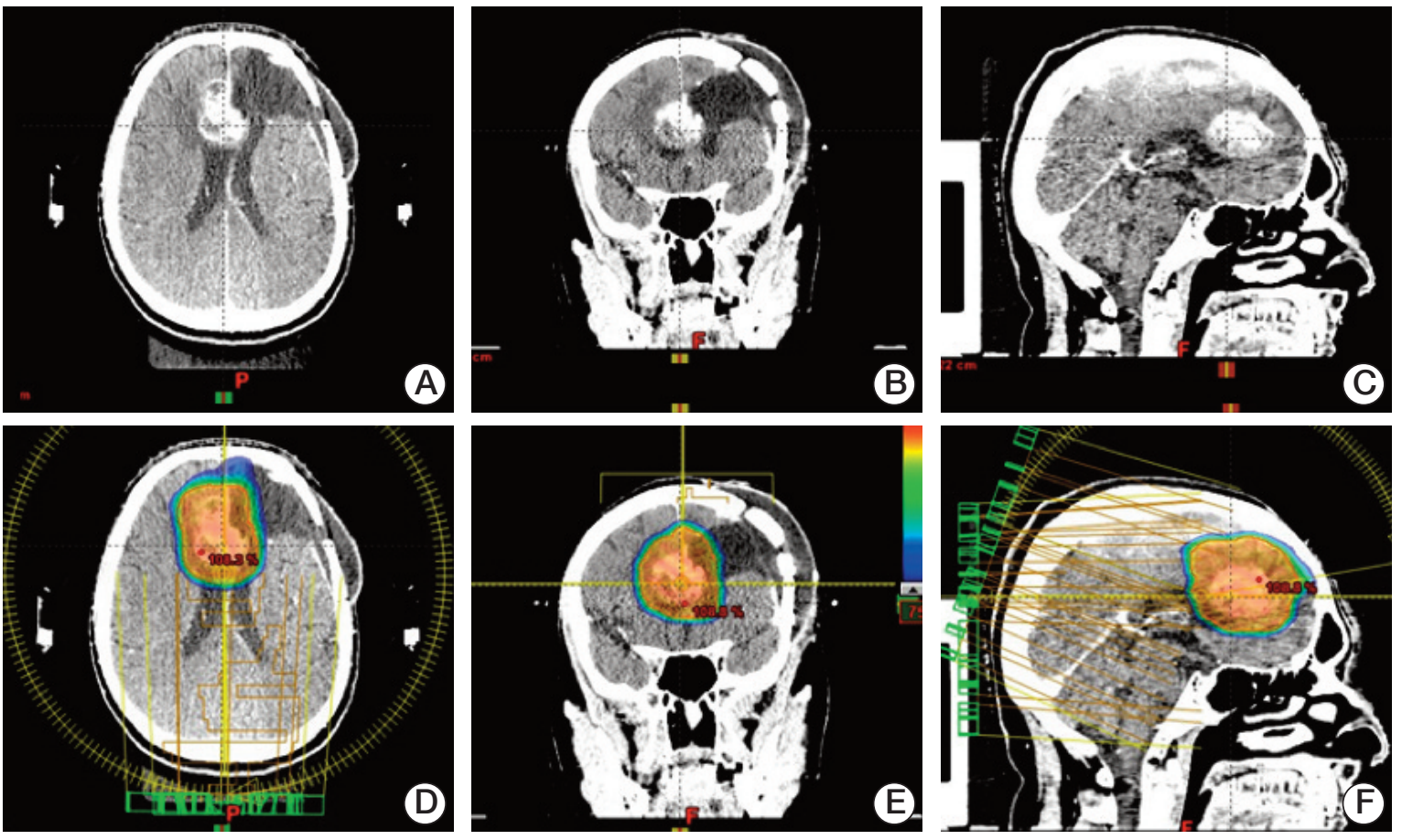

Fig. 1. Three views of the computed tomography scan showing the recurrence site (A-C) and dose distribution from the volumetric modulated arc therapy plan (D-F) for one patient.

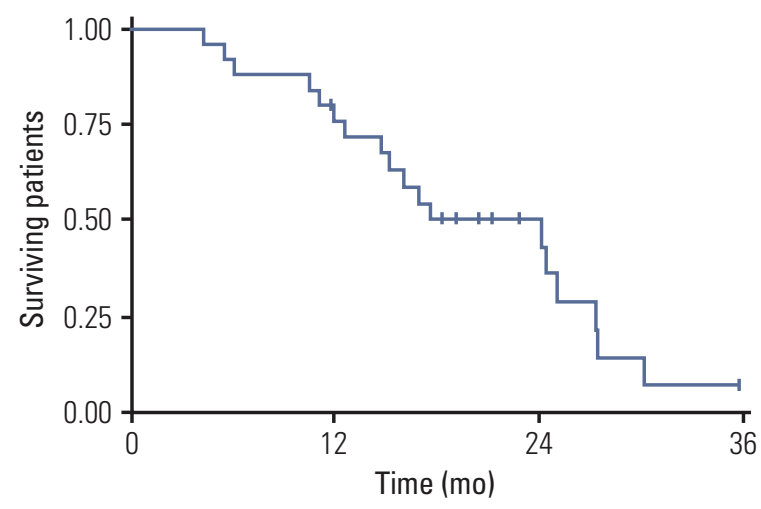

Fig. 2. Overall survival in high-grade glioma patients.

to the iPlan-net Brainlab stereotactic treatment planning system (Brainlab Ag, Feldkirchen, Germany) Automatic rigid co-registration, eventually manually corrected, was performed. The gross tumor volume (GTV) was defined as the tumor visible on enhanced T1-MRI, the clinical target volume (CTV) was generated by adding an isotropic margin of $5 \mathrm{~mm}$

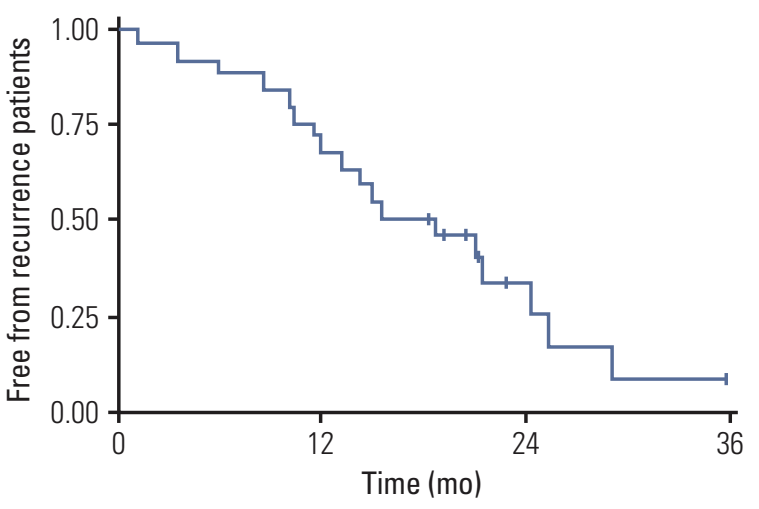

Fig. 3. Progression-free survival in high-grade glioma patients.

to GTV, and finally the planning target volume was obtained by expanding CTV of $3 \mathrm{~mm}$. Critical structures delineated were brainstem, optic nerves and chiasm. The dose prescription was 25 Gy in daily five fractions in almost all patients (with the exception of one patient that will be detailed in the results). Plans were processed using the RapidArc volumet- 
Table 3. Overall survival and progression-free survival stratified by histology and parameters

\begin{tabular}{|c|c|c|c|c|c|c|}
\hline Variable & 1-Year OS & 2-Year OS & p-value & 1-Year PFS & 2-Year PFS & p-value \\
\hline \multicolumn{7}{|l|}{ Diagnosis } \\
\hline GBM & 61 & 35 & 0.29 & 62 & 35 & 0.32 \\
\hline Grade III & 89 & 66 & & 83 & 18 & \\
\hline \multicolumn{7}{|c|}{ Results by EOR at diagnosis } \\
\hline Complete resection & 73 & 59 & $<0.01$ & 73 & 39 & $<0.01$ \\
\hline Partial resection & 86 & 43 & & 71 & 0 & \\
\hline \multicolumn{7}{|l|}{ Results by MGMT } \\
\hline Methylated & 100 & 83 & 0.05 & 100 & 83 & 0.08 \\
\hline Un-methylated & 67 & 67 & & 67 & 33 & \\
\hline \multicolumn{7}{|c|}{ Results by recurrent tumor volume } \\
\hline$\leq 50 \mathrm{~cm}^{3}$ & - & 56 & 0.26 & - & 42 & 0.1 \\
\hline$>50 \mathrm{~cm}^{3}$ & - & 33 & & - & 11 & \\
\hline \multicolumn{7}{|c|}{ Results by treatment approach } \\
\hline Combined & 83 & 51 & 0.89 & 74 & 32 & 0.94 \\
\hline RT alone & 73 & 0 & & 67 & 0 & \\
\hline
\end{tabular}

Values are presented as percentage. GBM, glioblastoma; EOR, extent of resection; MGMT, methylguanine-DNA methyltransferase; RT, radiotherapy.

ric modulated arc therapy (RA, Varian Medical System, Palo Alto, CA) to ensure maximal dose conformity and rapid dose fall-off towards critical structures as shown in Fig. 1. ExacTrac X-Ray 6D system and 3D cone-beam CT images were used for daily patient set-up and isocenter positioning.

\section{Outcome evaluation and statistical analysis}

Clinical outcome was evaluated by clinical neurological examination and brain MRI 1 month after treatment and then every 3 months. Response was recorded using the RANO criteria [22]. Hematologic and non-hematologic toxicities were graded according to Common Terminology Criteria for Adverse Events ver. 4.0.

Standard descriptive statistics (mean, standard deviation, and cross tabulation analysis) were used to describe the data's general behavior. Survival and recurrence time observations were plotted using Kaplan-Meier methods, starting from the date of recurrence. Univariate analysis was performed with the log-rank test to investigate the prognostic role of individual variables. A backward stepwise (probability to remove at 0.25 ) multivariate Cox regression model was used to estimate the independent associations of a variable set with overall/progression free survival. All analyses were sex and age adjusted.

\section{Results}

At diagnosis, all patients underwent surgery, radiotherapy with concomitant and adjuvant TMZ following the Stupp scheme. CR was obtained in 15 patients $(60 \%)$, SR in one patient $(4 \%), \mathrm{PR}$ in seven patients $(28 \%)$, and biopsy in two patients $(8 \%)$. The median interval time from the initial diagnosis to the recurrence was 19.27 months (range, 7 to 76 months) and 25 patients were re-irradiated.

Six patients underwent RT alone, while 19 patients underwent combined treatment with surgery and/or chemotherapy as shown in Table 2 . The mean recurrence tumor volume was $35 \mathrm{~cm}^{3}$ (range, 2.46 to $116.7 \mathrm{~cm}^{3}$ ). Twenty-four patients $(96 \%)$ received $25 \mathrm{~Gy}$ in five fractions except one patient who underwent a 50 Gy schedule due to young age and the small volume of the recurrence (well separated and distant from other structures at risk). No severe hematologic or neurologic toxicity was recorded during treatment and neurological examination scores remained stable. During follow-up increased antiepileptic drugs (AEDs) and corticosteroids were needed due to disease progression. At the last observation time, eight patients (32\%) were alive and 17 (68\%) were dead.

One-year OS was 76\% and 2-year 50\%, while 1-year progression-free survival (PFS) for the entire cohort of patients was $72 \%$ and at 2 years, 34\% (Figs. 2 and 3). The median PFS from recurrence was 16 months (range, 1 to 36 months) and OS was 18 months (range, 4 to 36 months). 
Table 4. Progression free survival: univariate and backward stepwise Cox multivariate regression

\begin{tabular}{|c|c|c|c|c|}
\hline \multirow{2}{*}{ Variable } & \multicolumn{2}{|c|}{ Univariate analysis } & \multicolumn{2}{|c|}{ Multivariate analysis } \\
\hline & Chi-square & p-value & HR $(95 \%$ CI) & p-value \\
\hline Histology & 0.97 & 0.32 & $1.39(0.39-4.98)$ & 0.61 \\
\hline Treatment & 0.01 & 0.94 & $0.88(0.18-4.19)$ & 0.87 \\
\hline EOR at diagnosis & 25.21 & $<0.001$ & $1.19(0.72-1.97)$ & 0.50 \\
\hline MGMT & 3.78 & 0.05 & - & - \\
\hline Volume & 2.03 & 0.15 & $0.98(0.95-1.01)$ & 0.23 \\
\hline
\end{tabular}

MGMT was not included in the multivariate analysis because of the limited number of available observations. HR, hazard ratio; $\mathrm{CI}$, confidence interval; EOR, extension of resection; MGMT, methylguanine-DNA methyltransferase.

Table 5. Overall survival: univariate and backward stepwise Cox multivariate regression

\begin{tabular}{lcccc} 
& \multicolumn{2}{c}{ Univariate analysis } & \multicolumn{2}{c}{ Multivariate analysis } \\
\cline { 2 - 5 } Variable & Chi-square & p-value & HR $(95 \%$ CI $)$ & p-value \\
Histology & 1.10 & 0.29 & $1.47(0.42-5.11)$ & 0.54 \\
Treatment & 0.02 & 0.89 & $0.94(0.20-4.49)$ & 0.94 \\
EOR at diagnosis & 24.65 & $<0.001$ & $1.12(0.68-1.83)$ & 0.66 \\
MGMT & 3.01 & 0.08 & - & - \\
Volume & 1.28 & 0.26 & $0.99(0.96-1.01)$ & 0.32 \\
\hline
\end{tabular}

MGMT was not included in the multivariate analysis because of the limited number of available observations. HR, hazard ratio; $\mathrm{CI}$, confidence interval; EOR, extension of resection; MGMT, methylguanine-DNA methyltransferase.

The results stratified by histology are summarized in Table 3 . On univariate and multivariate analysis neither gender, age, recursive partitioning analysis class, nor time of relapse were predictive of survival. In our series, the only factor influencing survival (in univariate analysis) was the EOR at initial diagnosis. The methylguanine-DNA methyltransferase promoter status showed a tendency to significance $(0.01<p$ $<0.05$ ) as shown in Tables 4 and 5.

Given the different prognosis of GBM patients compared to grade III gliomas, survival was also analyzed by histology. When GBM and grade III were studied separately, the only factors significantly impacting survival were, again, the use of combined treatment at recurrence compared to single modality of treatment $(\mathrm{p}<0.04)$ and the EOR at initial diagnosis $(\mathrm{p}<0.04)$.

Concerning toxicity, an increase of administration of steroids or AEDs was never required. No symptomatic radio-necrosis were observed. No other relevant symptoms of toxicity were observed.

\section{Discussion}

Available treatments for recurrent HGG include systemic therapy with $\mathrm{CT}$, local treatment such as re-irradiation and maximal safe resection, and of course, best supportive care. For recurrent tumors, the best treatment option is broadly debated, and the role of local treatment is an open question. The utility of RT in HGG glioma recurrence has recently been revisited, evaluating the effect of different modalities of dose delivery such as SRS, FSRT, or HSRT. Most authors recommend an interval of at least 6 months between the first and the second irradiation $[15,18,19]$ to allow repair of radiation damage [14]. The effects of re-irradiation alone are interesting and comparable to those of other single treatment modalities, with a median survival of 9 months and acceptable toxicity rates $[15,24,25]$. Recent studies show a satisfactory benefit with minimum toxicity of HSRT. The largest trial to date was performed by Fokas et al. [18] on 53 GBM patients who were re-irradiated using HSRT. Re-irradiation was well tolerated (no acute or late toxicity $>$ grade 2). Despite the relatively large median tumor volumes $(35.01 \mathrm{~mL})$, the median survival was nine months, and the 1-year PFS was $22 \%$. Ernst-Stecken et al. [19] evaluated efficacy, side effects 
and quality of life in patients who underwent HSRT with fractionation regiments of $7 \mathrm{~Gy}$ in five fractions. In this study the median tumor volume was $22.4 \mathrm{~cm}^{3}$ (range, 0.77 to 21.94 $\mathrm{cm}^{3}$ ), no severe toxicity was recorded, the PFS rate at one year was $53 \%$, and in two thirds of patients, quality of life measured by the European Organization for Research and Treatment of Cancer Quality of Life Questionnaire scores remained stable for a median time of nine months. Henke et al. [20] reported results of re-irradiation of two grade III and 29 grade IV tumors with hypo-fractionated radiotherapy up to $20 \mathrm{~Gy}$ (4 or $5 \mathrm{~Gy}$ per fraction). The median overall survival after re-irradiation was 10.2 months, and the median overall survival time after primary diagnosis was 30.9 months. No severe toxicity was observed.

In our series all patients underwent HSRT, in most cases combined with surgery and/or chemotherapy. Only six patients who were considered unfit for CT or refused CT received $\mathrm{RT}$ alone. The median time between the first and the second radiation treatment was 23 months (range, 9 to 93 months). The use of an hypo-fractionated scheme instead of a standard fractionation or radio-surgery was preferred for the following reasons (1) to reduce the treatment time with subsequent reduction of the patient discomfort; (2) to achieve the radiobiological advantage of fractionation; (3) to treat even large tumor volumes with low risk of acute or sub-acute toxicity; and (4) to reduce the incidence of symptomatic radio-necrosis. It is of note that in our series the median tumor volume was $35 \mathrm{~cm}^{3}$ (range, 2.46 to $116.7 \mathrm{~cm}^{3}$ ) and no relevant toxicity profiles were observed. Conversely, in cases treated with SRS, there is an increased risk of radio-necrosis and re-operation, up to $31 \%[17,20]$. Hall et al. [16], using an SRS based approach in relapsing HGG, documented high toxicity related to this approach. Thirty-five patients with large (median treatment volume, $28 \mathrm{~cm}^{3}$ ) recurrent tumors (74\% GBM, 26\% anaplastic astrocytoma) were treated. Seven patients required surgical resection for increasing mass, indicative of radiation necrosis after radio-surgery (mean, 4.0 months). Thus an actuarial re-operation rate of $31 \%$ was seen in this series. However, at present, a clear volume cutoff cannot be deduced from the literature. Thus, based on the reported toxicity, patient selection has to be performed very cautiously. No clear consensus is available on the optimal total dose to deliver or kind of fractionation to use. The available published data on normal brain radio-necrosis suggest defining the cumulative normalized total doses (cNTD) to evaluate the risk of healthy brain structures damage.

In our series, all patients received $25 \mathrm{~Gy}$ in five fractions except one patient who underwent $50 \mathrm{~Gy}$ in 10 fractions. We choose to utilize this schedule in most patients based upon the cNTD (103.75), the large recurrent volume treated (median volume, $35 \mathrm{~cm}^{3}$ ), and in some cases the close proximity of critical structures. We noted no experience of symp- tomatic radio-necrosis.

The main limitations of the present study are the retrospective nature, the heterogeneity of clinical characteristics, including both GBM and grade III gliomas, treatment variations, and the lack in some cases of bio-molecular features. Nevertheless, we feel that some interesting suggestions can be drawn. As expected, histology affected survival, with GBM patients having a worse outcome compared to grade III glioma. Unfortunately, 1p19 q codeletion, IDH1 mutation, age, and KPS did not affect survival. This is probably due to the small sample and low statistical power. With small and heterogeneous series, however, usually no statistically significant conclusion can be drawn. Despite that, patients who received greater advantage from re-irradiation were those who underwent CR at initial diagnosis. Particularly, no patients who underwent SR, PR, or biopsy at diagnosis were alive at 2 years after re-irradiation, nor were patients who underwent re-irradiation alone. The advantage of a combined treatment approach has already emerged but no prospective comparative study has been published yet.

\section{Conclusion}

This study suggests that HSRT could be a safe and feasible therapeutic option for recurrent HGG even in patients with larger tumors. A multidisciplinary evaluation should be mandatory to assure the best treatment for selected patients and local treatment should be considered as part of an integrated approach. The time is ripe to organize prospective cooperative studies.

\section{Conflicts of Interest}

L. Cozzi is Clinical Research Scientist at Humanitas Cancer Center and acts as Scientific Advisor at Varian Medical Systems AG (Cham, Switzerland). 


\section{References}

1. Stupp R, Hegi ME, Mason WP, van den Bent MJ, Taphoorn MJ, Janzer RC, et al. Effects of radiotherapy with concomitant and adjuvant temozolomide versus radiotherapy alone on survival in glioblastoma in a randomised phase III study: 5-year analysis of the EORTC-NCIC trial. Lancet Oncol. 2009; 10:459-66.

2. National Comprehensive Cancer Network. NCCN guidelines: central nervous system cancers 2012 [Internet]. Fort Washington, PA: National Comprehensive Cancer Network; 2012 [cited 2014 Dec 1]. Available from: http://www.nccn.org/ professionals / physician gls/f/guidelines.asp.

3. Minniti G, Amelio D, Amichetti M, Salvati M, Muni R, Bozzao A, et al. Patterns of failure and comparison of different target volume delineations in patients with glioblastoma treated with conformal radiotherapy plus concomitant and adjuvant temozolomide. Radiother Oncol. 2010;97:377-81.

4. Garden AS, Maor MH, Yung WK, Bruner JM, Woo SY, Moser $\mathrm{RP}$, et al. Outcome and patterns of failure following limitedvolume irradiation for malignant astrocytomas. Radiother Oncol. 1991;20:99-110.

5. Hess CF, Schaaf JC, Kortmann RD, Schabet M, Bamberg M. Malignant glioma: patterns of failure following individually tailored limited volume irradiation. Radiother Oncol. 1994;30: 146-9.

6. Barbagallo GM, Jenkinson MD, Brodbelt AR. 'Recurrent' glioblastoma multiforme, when should we reoperate? Br J Neurosurg. 2008;22:452-5.

7. Park JK, Hodges T, Arko L, Shen M, Dello Iacono D, McNabb A, et al. Scale to predict survival after surgery for recurrent glioblastoma multiforme. J Clin Oncol. 2010;28:3838-43.

8. Wick W, Platten M, Weller M. New (alternative) temozolomide regimens for the treatment of glioma. Neuro Oncol. 2009;11:69-79.

9. Wick W, Weller M, Weiler M, Batchelor T, Yung AW, Platten M. Pathway inhibition: emerging molecular targets for treating glioblastoma. Neuro Oncol. 2011;13:566-79.

10. Combs SE, Gutwein S, Thilmann C, Huber P, Debus J, SchulzErtner D. Stereotactically guided fractionated re-irradiation in recurrent glioblastoma multiforme. J Neurooncol. 2005;74: $167-71$.

11. Combs SE, Widmer V, Thilmann C, Hof H, Debus J, SchulzErtner D. Stereotactic radiosurgery (SRS): treatment option for recurrent glioblastoma multiforme (GBM). Cancer. 2005;104: 2168-73.

12. Biswas T, Okunieff P, Schell MC, Smudzin T, Pilcher WH, Bakos RS, et al. Stereotactic radiosurgery for glioblastoma: retrospective analysis. Radiat Oncol. 2009;4:11.

13. Fuller CD, Choi M, Forthuber B, Wang SJ, Rajagiriyil N, Salter $\mathrm{BJ}$, et al. Standard fractionation intensity modulated radiation therapy (IMRT) of primary and recurrent glioblastoma multiforme. Radiat Oncol. 2007;2:26.

14. Ang KK, Price RE, Stephens LC, Jiang GL, Feng Y, Schultheiss $\mathrm{TE}$, et al. The tolerance of primate spinal cord to re-irradiation. Int J Radiat Oncol Biol Phys. 1993;25:459-64.

15. Combs SE, Debus J, Schulz-Ertner D. Radiotherapeutic alternatives for previously irradiated recurrent gliomas. BMC Cancer. 2007;7:167.

16. Hall WA, Djalilian HR, Sperduto PW, Cho KH, Gerbi BJ, Gibbons JP, et al. Stereotactic radiosurgery for recurrent malignant gliomas. J Clin Oncol. 1995;13:1642-8.

17. Kong DS, Lee JI, Park K, Kim JH, Lim DH, Nam DH. Efficacy of stereotactic radiosurgery as a salvage treatment for recurrent malignant gliomas. Cancer. 2008;112:2046-51.

18. Fokas E, Wacker U, Gross MW, Henzel M, Encheva E, Engenhart-Cabillic R. Hypofractionated stereotactic reirradiation of recurrent glioblastomas: a beneficial treatment option after high-dose radiotherapy? Strahlenther Onkol. 2009;185:235-40.

19. Ernst-Stecken A, Ganslandt O, Lambrecht U, Sauer R, Grabenbauer G. Survival and quality of life after hypofractionated stereotactic radiotherapy for recurrent malignant glioma. J Neurooncol. 2007;81:287-94.

20. Henke G, Paulsen F, Steinbach JP, Ganswindt U, Isijanov H, Kortmann RD, et al. Hypofractionated reirradiation for recurrent malignant glioma. Strahlenther Onkol. 2009;185:113-9.

21. Fogh SE, Andrews DW, Glass J, Curran W, Glass C, Champ C, et al. Hypofractionated stereotactic radiation therapy: an effective therapy for recurrent high-grade gliomas. J Clin Oncol. 2010;28:3048-53.

22. Wen PY, Macdonald DR, Reardon DA, Cloughesy TF, Sorensen AG, Galanis E, et al. Updated response assessment criteria for high-grade gliomas: response assessment in neurooncology working group. J Clin Oncol. 2010;28:1963-72.

23. Sanai N, Polley MY, McDermott MW, Parsa AT, Berger MS. An extent of resection threshold for newly diagnosed glioblastomas. J Neurosurg. 2011;115:3-8.

24. Amelio D, Amichetti M. Radiation therapy for the treatment of recurrent glioblastoma: an overview. Cancers (Basel). 2012; 4:257-80.

25. Easaw JC, Mason WP, Perry J, Laperriere N, Eisenstat DD, Del Maestro R, et al. Canadian recommendations for the treatment of recurrent or progressive glioblastoma multiforme. Curr Oncol. 2011;18:e126-36. 